\title{
Una ciudadela estilo Recuay en el valle de Chacas (Perú): el sitio Pinchay-Riway. Una nota preliminar
}

Laura Laurencich-Minelli, Alberto Minelli y Carolina Orsini

\author{
CpenEdition \\ Journals \\ Edición electrónica \\ URL: http://journals.openedition.org/jsa/1890 \\ DOI: $10.4000 /$ jsa. 1890 \\ ISSN: 1957-7842 \\ Editor \\ Société des américanistes
}

Edición impresa

Fecha de publicación: 1 enero 2001

Paginación: 325-338

ISSN: 0037-9174

Referencia electrónica

Laura Laurencich-Minelli, Alberto Minelli y Carolina Orsini, « Una ciudadela estilo Recuay en el valle de Chacas (Perú): el sitio Pinchay-Riway. Una nota preliminar », Journal de la société des américanistes [En línea], 87 | 2001, Publicado el 17 noviembre 2005, consultado el 20 abril 2019. URL : http:// journals.openedition.org/jsa/1890; DOI : 10.4000/jsa.1890 


\title{
UNA CIUDADELA ESTILO RECUAY \\ EN EL VALLE DE CHACAS (PERÚ) \\ EL SITIO PINCHAY-RIWAY : UNA NOTA PRELIMINAR ${ }^{1}$
}

\author{
Laura LAURENCICH-MINELLI*, Alberto MINELLI**, \\ Carolina ORSINI*
}

Poco se sabe del valle de Chacas (Provincia de Asunción, Dep. de Ancash, Perú), desde el punto de vista de la arqueología, por haber sido, durante muchos años, zona peligrosa a causa del movimiento guerillero de "Sendero Luminoso ». Afortunadamente la situación ha cambiado recientemente y desde hace unos años están empezando las actividades de recuperación y puesta en valor del patrimonio arqueológico de la zona, gracias a la fructuosa colaboración entre el INC peruano, la alcaldía de Chacas, la Parroquia local del padre salesiano Hugo de Censi, el Ministerio de Asuntos Exteriores italiano y la Universidad de Bolonia. En este contexto, se concibió la idea de realizar un detallado mapa arqueológico del valle, comenzando con el levantamiento del sitio de Pinchay-Riway. Aquí se relatan los resultados de la campaña de topografía y arqueología de superficie del año 1999 con referencia al sitio de Pinchay-Riway.

\section{El Sitio Pinchay-Riway}

El sitio, que hemos denominado Pinchay-Riway, se encuentra en la vertiente oriental de la Cordillera Blanca y corresponde al cerro Riway $\left(9^{\circ} 09^{\prime} 83^{\prime \prime}\right.$ de latitud Sur y $77^{\circ} 24^{\prime} 18^{\prime \prime}$ de longitud Oeste); domina hacia el N-NE el valle de Chacas mientras que, hacia el S-SE, es dominado por los nevados de los cerros Huaychojirca y Cochahuaracanan. El pueblo más cercano es Sapchá que se encuentra a una hora de camino.

El sitio se puede dividir en dos zonas, A y B. La primera zona, es decir la A, está amurallada y corresponde a un alto espolón caracterizado por dos cerritos cuyas cumbres fueron allanadas para formar dos plazuelas circulares. De las susodichas plazuelas la primera se encuentra a 4009 m. s.n.m. y la segunda tres metros más

\footnotetext{
* Università di Bologna, Dip. Di Paleografia e Medievistica, Piazza S. Giovanni Monte 2, 40124 Bologna.

** Università di Ancona, Dip. DIBIAGA, 60100 Anconna.
}

Journal de la Société des Américanistes, 2001, 87 : p. 325 à 338. Copyright $\odot$ Société des Américanistes. 
arriba, es decir a 4012 m. s.n.m. Los dos cerritos están unidos por un horcajo natural. La superficie de la zona A es de $5694 \mathrm{~m}^{2}$ y la vegetación es herbácea (ichu). La muralla incluye las rocas del espolón y se alza apenas unos $50 \mathrm{~cm}$ sobre el nivel de los antiguos pisos de la que, a la lectura arqueológica, resulta ser una ciudadela amurallada. Al interior de la muralla, los edificios están actualmente derrumbados : sin techo y con los muros internos y externos arruinados.

La segunda parte, es decir la zona B, corresponde a un pequeño valle bien abrigado de la vertiente sur y dominado por el espolón amurallado : las ruinas allí descienden hacia un manantial (3966 m. s.n.m) y están delimitadas y conectadas con la zona A por una muralla discontinua y ahora incompleta, que no tenía la función de contención. Muralla que delínea una superficie de unos $15000 \mathrm{~m}^{2}$ en donde la vegetación es también herbácea (ichu). Aquí se halla el único edificio todavía techado: un montículo-tumba.

Ruinas hay también en la vertiente que se abre al norte del espolón y mira hacia el valle de Chacas : están en un terreno cuya pendiente es más suave y baja hasta los 3800 m. s.n.m. ; allí no se observaron muros de delimitación. La zona B cubre unos $20000 \mathrm{~m}^{2}$ e incluye un manantial; hoy en día allí hay cultivos de lupinos y papas. Dicha zona parece haber sido lugar de habitaciones esparcidas cuyos muros han sido saqueados para material de construcción, como desplazados para preparar el terreno para los cultivos. Por lo tanto, resulta difícil reconocer los antiguos edificios sin haber realizado excavaciones arqueológicas.

\section{IDENTIFICACIÓN DE LAS CONSTRUCCIONES Y SUBDIVISIÓN DEL SITIO EN SECTORES}

Las ruinas de Pinchay-Riway muestran muros de piedras y presentan planta ya sea cuadrada/rectangular/trapezoidal, ya sea circular: lo que nos permitió identificar cercos de contención/defensa, edificios y tumbas. Los cercos de contención son múltiples donde el declive es muy fuerte.

En armonía con las estructuras reconocidas gracias al trabajo arqueológico de superficie y de acuerdo con la morfología del terreno, se identificaron, en PinchayRiway varios sectores que aquí se presentan (Figura 1).

En la zona A, es decir en la ciudadela amurallada, se identificaron los siguientes sectores :

- Primer sector ${ }^{2}$ (3 993 m. s.n.m.), (Figuras 2a y 2b) : sector de acceso controlado a la ciudadela, mediante un pasadizo/puerta constituido por dos muros paralelos interrumpidos de manera desplazada uno con respecto al otro. De acuerdo con lo que se ve hoy, este pasadizo habría permitido el control de la entrada de la ciudadela. Don Fidencio Portella, un anciano de Sapchá, afirma que, antes del temblor de 1970, aquí había lo que llama una torre. Desafortunadamente no hallamos fotos que lo comprueben, pero la importancia de los muros de las habitaciones la, 1b, 1c, atestigua que hubo una construcción de tres piezas más alta que las otras, bien acabada, y con unas hornacinas en el interior (Figura 3b). Dicha construcción se abre hacia los patios 1d y 1e (Figura 1).

- Segundo sector (3 994 m. s.n.m.) : sector habitacional de la élite, lo indica el acabado de las paredes de las habitaciones, unas de las cuales presentan también 


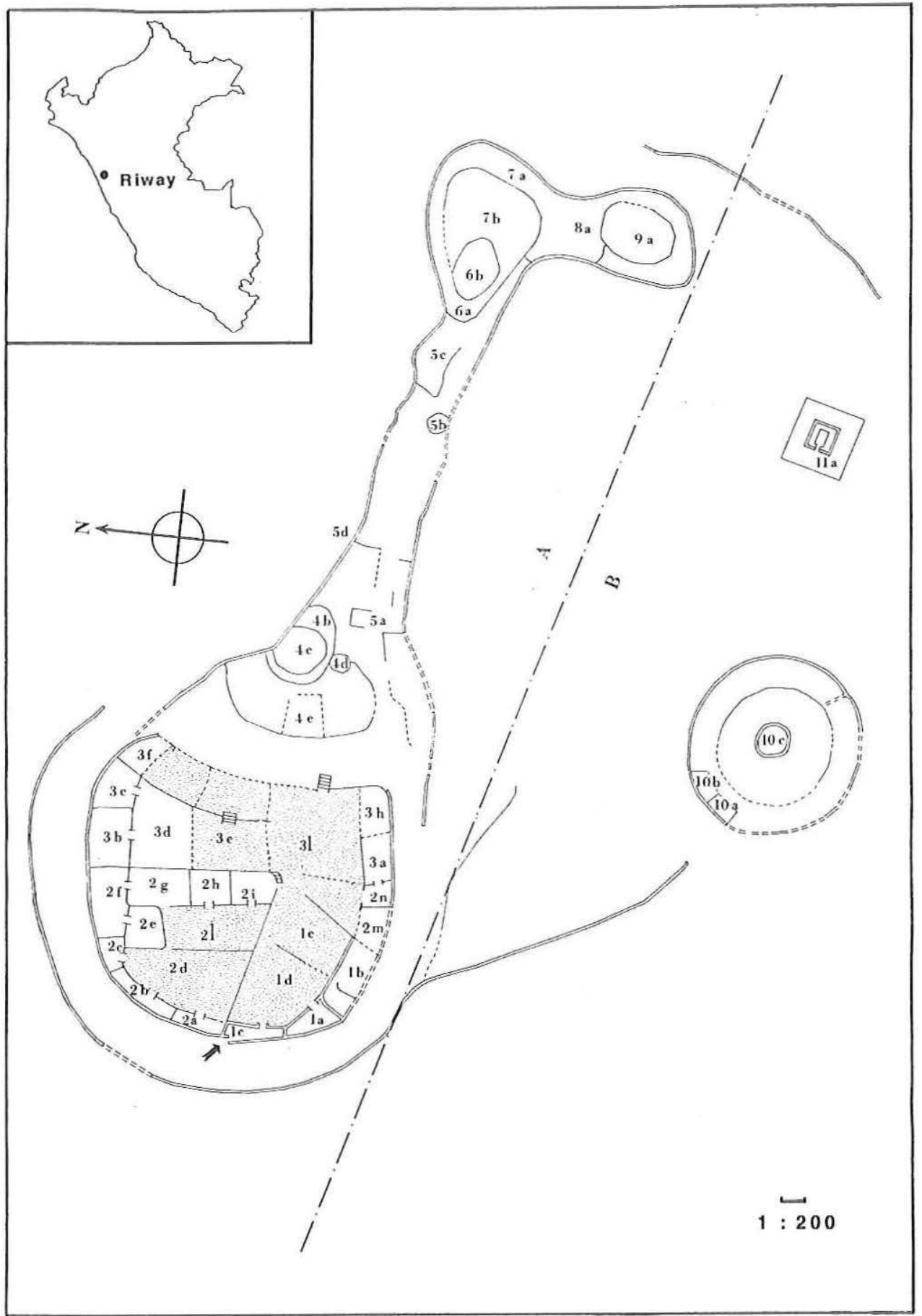

Fig. 1. - Croquis topográfico del sitio Pinchay-Riway. La recta rasgueada separa el sector A del sector B. Los números indican los sectores y las letras indican las estructuras. Las líneas dobles indican los muros de doble cara, las líneas finas los muros sencillos, los espacios punteados los patios 

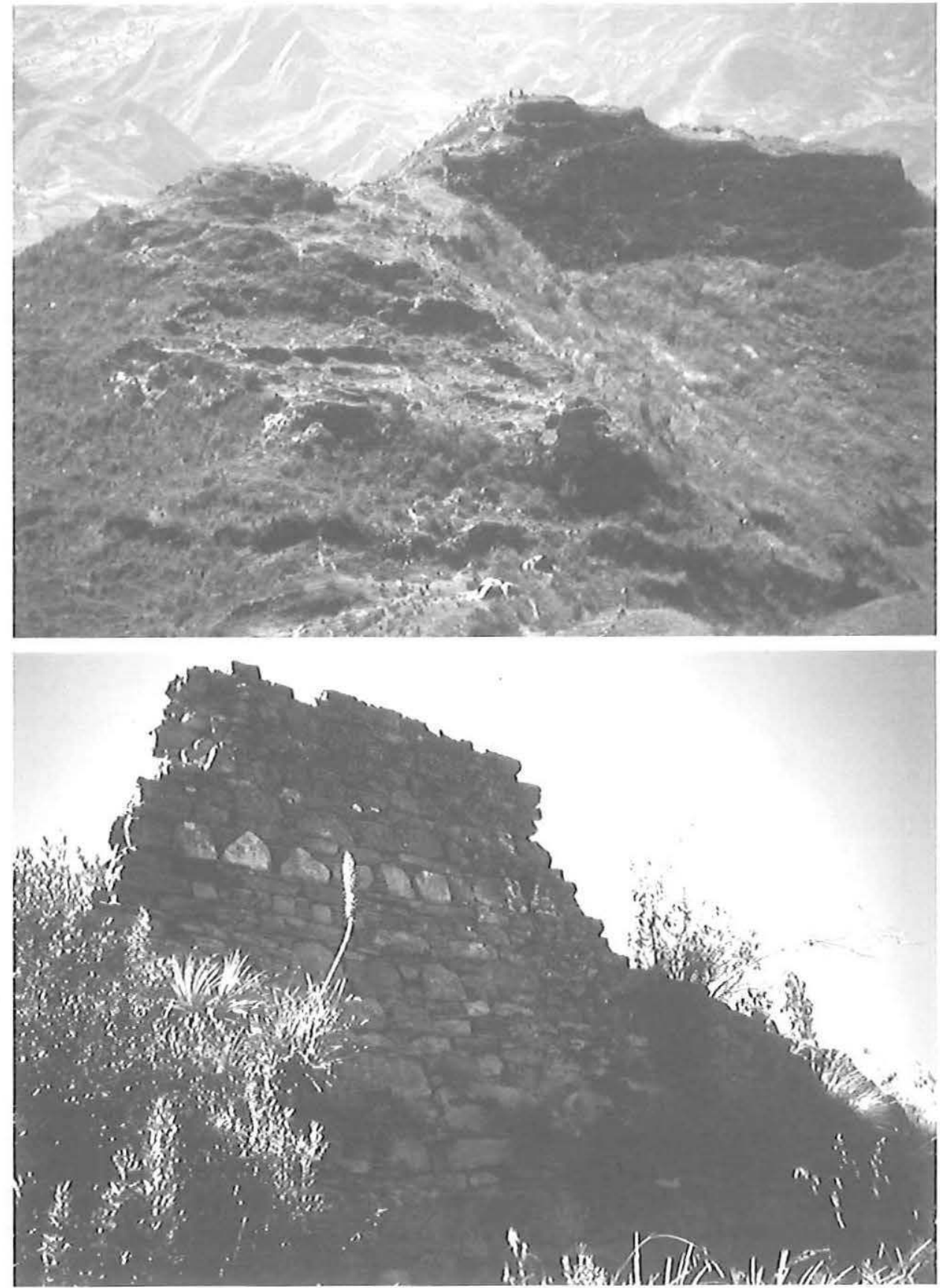

Fig. 2 .

a. Vista del sitio de Pinchay-Riway : se detectan los sectores 1, 2, 3, los dos pirushtu (sect. 4 y 6) y las plazoletas cementeriales (sect. 7,8,9)

b. Muro de doble cara de la entrada del sect. 1 
hornacinas. Se identifican dos habitaciones grandes : una formada por tres piezas (2a, $2 \mathrm{~b}, 2 \mathrm{c})$ que dan a un patio (2d) y la otra formada por cinco piezas $(2 \mathrm{e}, 2 \mathrm{f}, 2 \mathrm{~g}, 2 \mathrm{~h}, 2 \mathrm{i})$ que abren al patio $2 \mathrm{l}$. Además, hay viviendas más pequeñas ( $2 \mathrm{~m}$ y $2 \mathrm{n})$.

- Tercer sector (3 997 m. s.n.m.) : sector habitacional más alto que el precedente, del cual está separado por un muro de contención que al mismo tiempo forma el lado oeste de los edificios y del patio 31 de este sector. Unas gradas de piedra llevan desde el segundo sector hacia el patio $3 \mathrm{l}$ del tercero. Los muros de las habitaciones aquí no presentan hornacinas, el acabado está menos pulido que en los del segundo sector y las viviendas presentan, cada una, una habitación única. Se observan trazas de reutilización en la vivienda 3a cuya entrada, hecha con tres monólitos (dos jambas y un dintel) fue luego tapiada con piedras sin pulir.

- Cuarto sector (4 $000 \mathrm{~m}$. s.n.m.) : se trata del primer cerrito de la ciudadela que, de acuerdo con la tradición local, llamaremos pirushtu. Esta palabra indica un topónimo, el sitio Pirushtu cerca de Chacas ${ }^{3}$, formado por una antigua pirámide artificial tronco-cónica escalonada que posiblemente tuvo una función ceremonial. En efecto la importancia ceremonial de la plazoleta circular más alta de un pirushtu estilo Recuay está también evidenciada por la documentación plástica brindada por las vasijas de este estilo. En particular nos referimos a aquellas vasijas que retratan a un miembro de la élite durante ceremonias que ocurren en un espacio circular y alto (Willey 1971, p. 150, Larco Hoyle 1975, Figura 81, Lothrop 1964, p. 186, Lapiner 1976, Figura 415, Gero 1999, p. 37).

El cuarto sector domina los tres sectores precedentes. Está separado del tercero por un muro arqueado que contiene el primer andén del pirushtu, donde se observa la base de una pequeña vivienda cuadrada y un alto muro circular, es decir un pequeño edificio de planta circular (chullpa) (4d, 4e). A éste, según uno va subiendo, sigue el segundo andén, contenido por un muro que forma un cerco elipsoidal (4b); luego hay el tercer andén que forma el rellano más alto $(4009 \mathrm{~m}$. s.n.m. ) : es la cumbre del cerrito que forma una plazoleta artificial y circular, contenida ésta también por un muro (4c). Aquí no hay viviendas. En espera de ulteriores investigaciones arqueológicas, se considera este sector como de tipo ceremonial por la estructura pirushtu.

- Quinto sector (4 $002 \mathrm{~m}$. s.n.m.) : corresponde a la silla natural que une los dos cerritos. Está delimitado, en los lados norte y sur, por la muralla de contención del espolón. Presenta las bases de edificios, cuya planta es de ángulo recto o semicircular que, debido a su ubicación y su forma, interpretamos como almacenes. De manera preliminar; se interpreta todo el quinto sector como una zona de enlace, almacenamiento y pasaje entre los dos cerritos.

- Sexto sector (4 $010 \mathrm{~m}$. s.n.m.) : está separado del quinto sector por un muro que contiene un andén (6a). Además hay otro andén contenido por un muro que forma un cerco elíptico : es decir la plazoleta artificial o pirushtu más alto de la ciudadela (4 012 m. s.n.m. : 6b). Allí, tal vez, se alzaba el esbelto monolito tronco-piramidal en granito blanco, actualmente derrumbado en el quinto sector. Provisionalmente, se considera este sector como ceremonial por presentar la estructura pirushtu.

- Séptimo sector (4 $008 \mathrm{~m}$. s.n.m.) : es la continuación del pirushtu del sector 6, pero lo hemos considerado a parte por presentar varias tumbas huaqueadas, es decir 

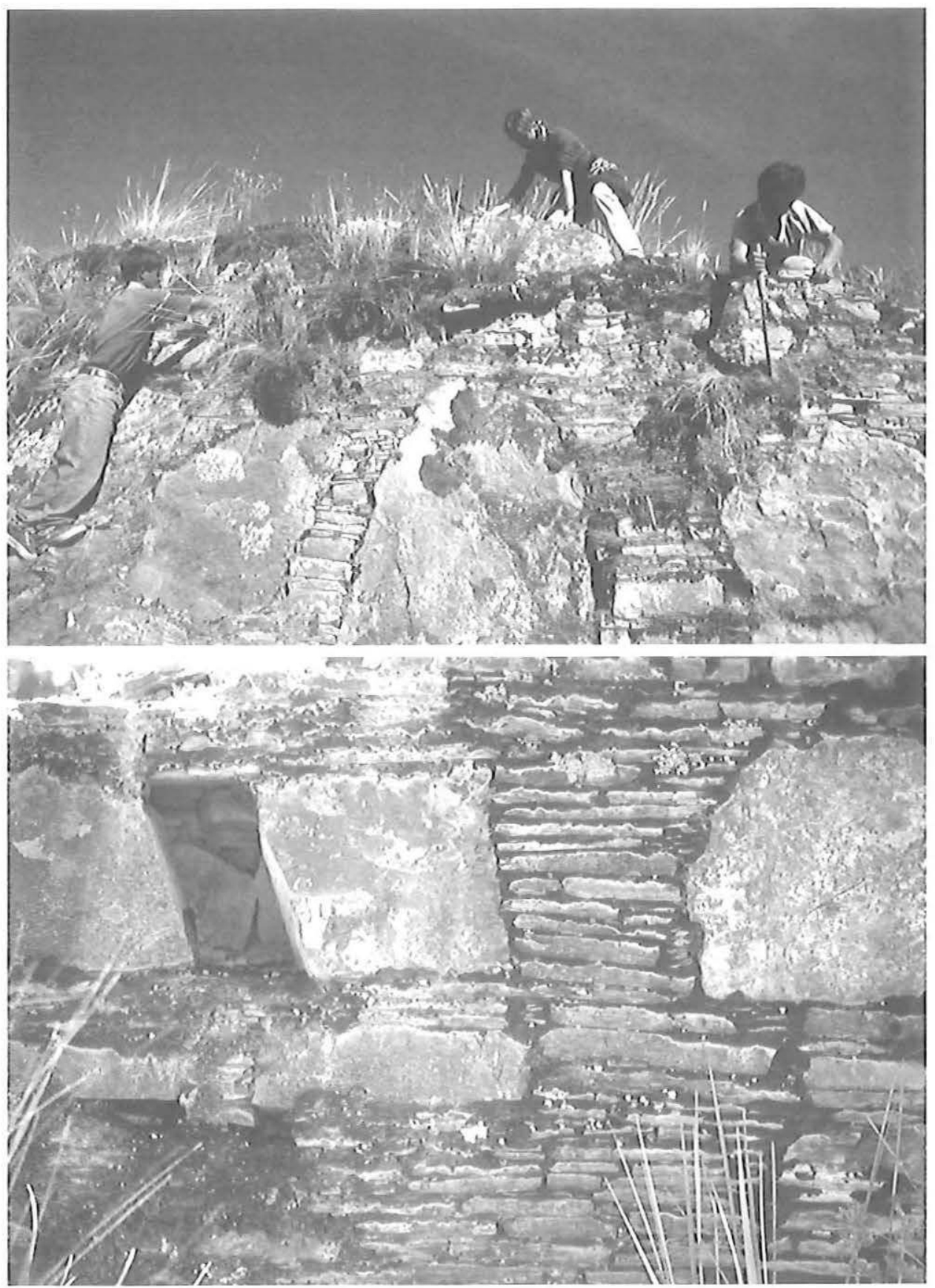

Fig. 3.

a. Muro de doble cara que encierra la ciudadela

b. Muro de doble cara de la pieza $\mathrm{lb}$, cara interna con una hornacina 
que se trata de un sector funerario. Está formado por dos andenes que suben al pirushtu del sector 6 : el más bajo es la continuación hacia el este del andén 6a, que aquí se denomina 7a, y el superior es el 7b. Como se ve en lo que dejaron los huaqueros, esta zona funeraria contenía tumbas hipogeas cuyos techos, hechos de grandes lajas, formaban el pavimento del sector.

- Octavo sector (4 $008 \mathrm{~m}$. s.n.m.) : plaza funeraria intermedia allanada en forma pseudo-circular (8a). Es un andén sostenido por un muro arqueado con dirección EO que lo separa de la plaza inferior, es decir del sector 9. Los otros dos lados de la plaza están contenidos por la muralla que rodea la ciudadela. Las grandes lajas del techo de las tumbas hipogeas saqueadas por los huaqueros, forman el pavimento de la plazoleta al igual que en el sector 7. No hay ruinas de viviendas.

- Noveno sector (4 $007 \mathrm{~m}$. s.n.m.) : plaza funeraria inferior allanada en forma circular. En los lados exteriores, está delimitada por la muralla que contiene el espolón. Internamente hay un muro circular discontinuo que delimita un cementerio elíptico (9a). Las lajas del techo de las tumbas hipogeas, saqueadas, aquí también formaban, al igual que en los sectores 7 y 8 , el pavimento de la plazoleta. No hay trazas de viviendas.

En el ámbito de la vertiente sur o zona B, se hallaron varias ruinas de piedras amontonadas $\sin$ orden que no permiten un reconocimiento topográfico preciso sin previas excavaciones arqueológicas que permitan detectar las eventuales bases de las construcciones. Por lo tanto y en espera de dichas excavaciones arqueológicas se identificaron apenas dos sectores (el décimo y el undécimo) donde las ruinas permiten una clara lectura.

— Décimo sector (3962 m. s.n.m.) : formado por tres cercos circulares y concéntricos, cada uno delimitado por un muro. Mientras que el muro externo es también de contención, los dos muros internos sirven únicamente para delimitar el espacio, es decir para formar las dos coronas del círculo y la plazoleta central que está hundida de $40 \mathrm{~cm}$ con respecto al piso de frecuentación de la corona misma (Figura 2). En el centro de la plazoleta se encuentran dos pocitos empedrados, abiertos uno a la par del otro (se describen más adelante). Tal vez se trate de pocitos para ofrendas (desafortunadamente ahora están saqueados). En la corona formada por los dos muros más externos hay trazas de pequeñas construcciones $(10 a, 10 b)$. Se interpreta este sector como de tipo ceremonial porque, en su parte más importante, es decir la interna, 1) no hay habitaciones, 2) la plazoleta central hundida presenta en su centro lo que, en el trabajo arqueológico de superficie, se interpretó como pozos de ofrendas.

Existen restos de un muro que conectaba este sector con la muralla de la ciudadela casi para unirlo a ella.

— Undécimo sector (3 970 m. s.n.m.) : tumba de la élite en montículo (se describe más adelante). Está rodeada por un muro cuadrado. La tumba, ya saqueada, presenta una planta cuadrada y la entrada da al oeste.

\section{TIPOLOGía DE LOS MUROS}

En Pinchay-Riway se hallaron tres clases de muros : muros de doble cara, muros sencillos, muros en seco. 


\section{Muros de doble cara}

Estos muros están formados por dos paredes paralelas de piedras pulidas (hechas mediante hileras de piedra gris amarillento) alternadas con hileras de piedras más pequeñas, gris oscuro, de estructura laminar, unidas con argamasa ; piedras que sirven también para tapar los intersticios entre las piedras grandes. La anchura de los muros de doble cara es muy variable : mide entre 90 y $200 \mathrm{~cm}$; sus rellenos internos están hechos con piedritas y argamasa (Figura 3a).

Dichos muros proporcionan un lindo efecto cromático y de volumen. Fueron utilizados para construir muros externos, sean éstos las murallas de contención/ defensa de la ciudadela o las paredes de edificios importantes. En el caso de las murallas de contención, la pared que mira hacia el desplome está construida, en la base, con piedras enormes gris amarillento que, según se eleva el muro, van reduciendo su tamaño. Los intersticios entre dichas piedras son rellenados con piedras laminares gris más oscuro que están pegadas con argamasa (Figura 3a). La pared interna, bien pulida, está en cambio construida con piedras gris amarillento de tamaño casi constante ${ }^{4}$ y piedras laminares gris más oscuro. Cuando el muro de doble cara está empleado en la construcción de edificios, ambas paredes, la interna y la externa, se ven bien pulidas y equilibradas en el tamaño y en el color de las piedras.

\section{Muros sencillos}

Es muy frecuente también esta clase de muro: se trata de muros sencillos (no dobles) construidos con piedras talladas, bastante grandes, de color gris (que a veces coge matices amarillento) cuyos intersticios están tapados con piedras más pequeñas, gris más oscuro de estructura laminar que están unidas con argamasa. Estos muros están pulidos pero no hay una búsqueda de juego de colores y de volumenes como en los muros de doble cara. La anchura de esta clase de muro varía entre 50 y $60 \mathrm{~cm}$. Los muros sencillos están empleados en construcciones de edificios y de murallas.

\section{Muros en seco}

El tercer tipo de muro que se halla en Pinchay-Riway, es el muro en seco, llamado pirca en el Perú : es un muro constituido solamente por las mismas piedras grises de los muros sencillos pero no son ni talladas, ni pulidas y se utilizaron sin argamasa. Están colocadas en dobles y triples hileras cruzadas entre ellas. Hemos hallado este tipo de muro en las construcciones de viviendas en la vertiente sur; en reconstrucciones de edificios ya hechos con muros sencillos (3a) y en la reutilización de partes de muros de defensa o de contención de la ciudadela (1c). En este caso puede tratarse de pircas más recientes.

\section{TRABAJO ARQUEOLÓGICO DE SUPERFICIE}

Una primera revisión superficial del sitio no nos permitió encontrar fragmentos cerámicos debido a que, después del corte de la vegetación y de la limpieza del suelo, había quedado una consistente capa de humus por encima del antiguo piso de ocupación. Otro impedimento a la búsqueda de la cerámica fue el hallazgo de 
multiples piedras, antes empleadas en la construcción de los edificios, ahora derrumbadas en la cercanía de los mismos. Como fue nuestra preocupación no alterar la interpretación de las posibles causas de los derrumbes, no quisimos mover las piedras derrumbadas para hacer allí el trabajo arqueológico de superficie.

Elegimos entonces revisar algunos pozos que ya habían sido saqueados por los huaqueros en el pasado.

De los 25 pozos de huaqueros detectados (cuya mayoría se encuentra en las tres plazas cementeriales) seleccionamos siete para una investigación detallada. La recolección de la cerámica ha sido efectuada mediante la limpieza de las paredes de los pozos. Observando las calas abiertas por los huaqueros podemos afirmar que el piso de frecuentación se encuentra a 20-30 cm de profundidad, es decir un poco más abajo de lo que atestigua Víctor Ponte (2000) en sitios contemporáneos del Callejón de Huaylas. Además se observó que algunas habitaciones tenían, a este mismo nivel, el piso empedrado al igual que el de las plazoletas circulares o pseudo-circulares de los sectores 4, 6, 7, 8, 9 y el de la plazoleta hundida del sector 10 .

Los tiestos que recogimos fueron analizados en sus pasta, desgrasantes/ inclusiones, engobe, tipo de cocción, dibujos decorativos y, cuando fue posible, se reconstruyó el tipo de forma a la cual el fragmento pertenecía (tazones, ollas, botellas, cuencos, torteras). Los fragmentos recogidos presentan semejanzas con formas y decoraciones de los tipos cerámicos Recuay tardíos ${ }^{5}$, pero sólo excavaciones arqueológicas nos permitirán construir una seriación adecuada para este sitio.

Reconocimos los siguientes tipos cerámicos: rojo/anaranjado rústico (con muchas variantes, de un color claro hasta un rojo/marrón), negro fino reducido, negro grueso obtenido aplicando engobe con grafito, blanco de caolín, cerámica pintada en rojo sobre anaranjado (un tipo muy común), cerámica pintada en rojo y negro sobre crema, y en blanco sobre rojo, además en negro sobre crema con técnica al negativo (un tipo más raro). Se hallaron también torteras (cada una recortada de un tiesto) y material lítico (bolas, cinceles). Es decir materiales que, comparados con los guardados en el Museo Arqueológico de Ancash (Huaraz), nos permiten afirmar que el sitio pertenece a la cultura Recuay (200-600 d.C.)

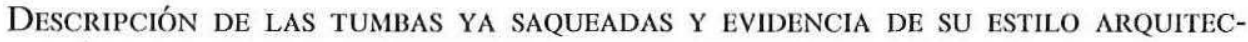 TÓNICO}

Los sectores octavo y noveno presentan respectivamente dos y catorce tumbas hipogeas saqueadas, tumbas cuyos techos fueron formados por las grandes lajas que constituyen el piso de las dos plazas. El trabajo de los huaqueros fue tan destructivo que ya no se logra leer la forma de las cámaras funerarias. En cambio, interesante ha sido la investigación en dos tumbas ya saqueadas, una en el sector 11 y la otra en el sector 5 , que aquí se describen porque los huaqueros no alteraron su arquitectura.

\section{Tumba $n^{\circ} 1$}

La tumba $n^{\circ} 1$ está ubicada en el sector 11 , es decir en los bajos de la vertiente sur y se presenta como un montículo tronco-cónico rodeado por un muro cuadrado de tipo sencillo. La limpieza que realizamos en un sondeo en la pared norte del montí- 
culo, puso de relieve que éste tiene planta cuadrada y está construido por un muro de doble cara, de $140 \mathrm{~cm}$ de altura, sobre el cual está puesto el techo construido según la tecnica de la falsa bóveda, es decir que se trata de una tumba en forma de casita con verdadera cámara funeraria interior. La entrada a la cámara es rectangular, formada por dos jambas y un dintel, de $65 \mathrm{~cm}$ de altura y de $50 \mathrm{~cm}$ de anchura y se abre al centro del muro del lado oeste del montículo. A través de un pasadizo de $60 \mathrm{~cm}$ de largo, se entra a la cámara funeraria : es rectangular ${ }^{6}$, construida con piedras pulidas y talladas (del tipo que hemos llamado muro sencillo) y adornada con hornacinas. El techo, construido en falsa bóveda, está parcialmente hundido en la parte centraloeste, mientras que está intacto en la parte este. Después del entierro, el muro de la tumba fue tapado con tierra formando un talud. Esto dio a la tumba el perfil tronco-cónico, la forma de montículo y al mismo tiempo escondió su entrada, probablemente para evitar eventuales saqueos. Hallamos varios fragmentos de cerámica negra y roja típica del sitio.

A pesar de tantas precauciones, los huaqueros encontraron la entrada a la tumba y la saquearon dejando en su interior apenas unos restos de huesos humanos disgregados, entre los cuales localizamos algunos que indican que allí había sido enterrado por lo menos un hombre adulto.

\section{Tumba $n^{o} 2$}

Una fisura natural en la roca, por debajo de la muralla que rodea el espolón en su lado norte (sector $5 \mathrm{~d}$ ), da a una pequeña cueva ${ }^{7}$ que es la antesala de una cámara funeraria. Allí encontramos unas ofrendas modernas (coca y flores). A pesar de que la tumba ha sido despojada de su ajuar, sigue siendo lugar de ofrendas por parte de la población local. Al oeste de la cueva-antesala, se ubica la entrada a la cámara funeraria : es baja con dos jambas y un dintel y mide $50 \mathrm{~cm}$ de altura y $35 \mathrm{~cm}$ de anchura. La cámara es rectangular y se prolonga hacia el oeste ${ }^{8}$. Allí están los huesos de dos personas adultas, una de sexo masculino y la otra probablemente de sexo femenino, inhumados pero actualmente muy deteriorados. Entre los huesos hay ofrendas vegetales modernas, tal vez dejadas por los huaqueros en cambio del ajuar fúnebre que robaron.

\section{DESCRIPCIÓN DEL SECTOR 10}

Muy interesante es el sector 10 , no sólo por su conservación relativamente buena sino también porque manifiesta el gusto por la búsqueda de la división del espacio en forma circular concéntrica. En efecto, caminando hacia el centro, se observa un muro circular externo de contención (muro de doble cara) cuya altura original, medida en las partes conservadas, era de 1,35 m (sobre el piso de frecuentación interno), luego otro muro (muro sencillo) concéntrico y de la misma altura, y un tercer muro (muro de doble cara bien acabado) concéntrico a los dos, de $1,50 \mathrm{~m}$ de altura cuyo piso está $40 \mathrm{~cm}$ más abajo que el piso de la corona ${ }^{9}$, es decir que se trata de una plazoleta circular hundida. En la primera corona, hay trazas de construcciones con pared arqueada del lado externo (estructuras 10a, 10b). En cambio es evidente que los dos muros circulares internos no contenían edificios. 
En el mismo centro del piso de la plazoleta hundida central y circular, hay dos pequeños pozos rectangulares, abiertos uno al lado del otro, cuyas paredes son empedradas y alcanzan la misma profundidad, es decir $40 \mathrm{~cm}^{10}$. Están separados por una laja que hace las veces de brocal de los pocitos y de pared interna. Las demás paredes están bien empedradas. Por su forma y ubicación, se interpretan como pozos de ofrenda. Resultan ya saqueados y se ve que estaban tapados por unas grandes lajas. Al limpiar las paredes de los pozos encontramos unos tiestos y, por encima de las lajas, un cincel bien pulido.

\section{REFLEXIONES PRELIMINARES}

La uniformidad de los tipos de muros y de los patrones constructivos (patrones que son aproximadamente cuadrados, de acuerdo con la topografía del terreno, o circulares) y los tiestos hallados indican que el sitio Pinchay-Riway fue construido en el marco de una única cultura, la Recuay. Se planeó el sitio encima de un espolón y se logró superar grandes problemas de estática al construir buena parte de la muralla de la ciudadela sobre precipicios y al realizar andenes que sostienen plazoletas circulares encima del cerro Riway. Para efectuar construcciones tan poderosas y movimientos de tierra tan complejos se debió de haber dispuesto de numerosos obreros y de una buena organización administrativa que no podía residir en Pinchay-Riway sino en un centro más grande. Se debía conocer, por intuición y por práctica, los principios basilares de la estática que se necesitan para realizar tales construcciones sobre los cerros, pero también algo de geometría para lograr planear cercos circulares concéntricos (como los del sector 10). Tal vez se ideó el sitio en escala, construyendo primero una maqueta en piedra (parecida a aquellos trabajos líticos que miden aproximadamente $50 \times 40 \times 20 \mathrm{~cm}$ y que se conocen como « juego o maqueta » y se hallan en el mismo valle de Chacas).

Es curioso que en Pinchay-Riway no encontramos monolitos grabados de estilo Recuay : puede ser o que los huaqueros ya se los llevaron o que no se quiso o no se pudo utilizar aquí esta forma de expresión. Por el contrario, en la ciudad moderna de Chacas, varios monolitos grabados de estilo Recuay o no, han sido reempleados como adornos en construcciones, monolitos que, según se dice, proceden del sitio de Pirushtu, ubicado en las alturas que rodean Chacas, y que plantean la discusión si este sitio ha sido utilizado también por los Recuay.

Es posible que el sitio de Pinchay-Riway fuera reutilizado en tiempos más tardíos, como lo indicaría el reempleo de muros de doble cara con pircas y el cierre de la entrada de una habitación del sector 3 (estructura 3a) también con pirca, mientras que la habitación está construida por un muro del tipo que calificamos de sencillo. Este trabajo de superficie no permitió identificar cuando tuvo lugar esta reutilización, pero hicimos la hipótesis de que fuera durante un período de decadencia cuando ya no se sabía o no se podía construir con las técnicas refinadas de los muros de doble cara o sencillos.

Sea lo que sea, la utilización del sitio sigue en el presente, aunque de manera muy esporádica, todavía hoy. En efecto recordamos que se encontraron ofrendas recientes tanto en la tumba $\mathrm{n}^{\circ}$ 2, como en la cumbre del pirushtu más alto, es decir en la 
plazoleta 6b. Según don Ramón Rojas de Chacas, gente del valle sube a PinchayRiway de vez en cuando para dejar ofrendas a « sus antepasados ».

Los trabajos realizados en Pinchay-Riway plantean muchas otras preguntas, en particular :

1. ¿Cuándo, durante la cultura Recuay, fue construida la ciudadela y cuánto tiempo fue ocupada?

2. ¿Cuál es la razón de la división del sitio entre un espolón amurallado a los 4000 m. s.n.m. y una zona baja, la zona B, en la vertiente sur; bien protegida?

3. ¿Por qué tantas tumbas y por qué tanta variación entre ellas (tumba-montículo, tumba-hipogea, tumba en una cueva), a pesar de un mismo estilo de construcción en la cámara mortuoria ?

En espera de que excavaciones arqueológicas sistemáticas vengan a aclarar todas estas preguntas, formularemos unas hipótesis de trabajo comparando los datos recabados con los de los sitios Recuay más conocidos, por ejemplo Pashash (Grieder 1978), Marenayoc, Quitapampa, Auquis Marca (Valladolid 1990, p. 10). De esta comparación resalta que el sitio Pinchay-Riway del valle de Chacas, a pesar de asemejarse a los asentamientos del Callejón de Huaylas en la cerámica y en las características arquitectónicas generales, parece tener sus propias particularidades. Entre ellas : 1) la ubicación en un alto cerro, 2) la urbanización más compacta y amurallada, la existencia de plazoletas circulares - pirushtu - en las cumbres y de una plazoleta circular hundida. Al mismo tiempo hay que tener presente que el cuadro de la cultura Recuay en el mismo Callejón de Huaylas se está modificando con las investigaciones que Ponte (2000) está realizando en los asientamientos elevados de dicho Callejón. El describe allí también dos pirámides tronco-cónicas unidas por un horcajo pero no describe sitios amurallados : por lo tanto podríamos decir que hay correspondencia estilística entre los sitios elevados del Callejón de Huaylas y PinchayRiway en lo que concierne a los dos pirushtu y al sector 5 .

Pinchay-Riway proporciona nuevos datos sobre el patrón urbanístico de altura de la cultura Recuay, no sólo en el valle de Chacas sino también en general, y abre el problema sobre los principios estilísticos-culturales que obligaban a incluir, en el patrón constructivo Recuay, los altos cerros y el valle que está debajo, pero respetándolos y amoldándolos de acuerdo con su proprio ideal cultural que parece haber sido de construir plazoletas circulares ceremoniales tanto en las cumbres como en el valle. En el primer caso se allanó la cumbre de las colinas y se sostuvo las plazoletas con andenes, en el segundo se abrió una plazoleta circular hundida.

Este sitio plantea también el problema de la organización política de los Recuay y el del significado que tenía en la cultura Recuay la división circular del espacio, como la de las plazoletas encima de los pirushtu y lo de la plazoleta hundida. Esta última, con sus pozos de ofrenda, puede haber sido relacionada con un culto a la diosa de la tierra (algo como la Pachamama de los tiempos incaicos). Recuerda, en su forma circular, una plazoleta hundida más antigua pero geográficamente no muy distante : la plazoleta de Chavín de Huantar. En cualquier caso la documentación plástica, brindada por las vasijas estilo Recuay, nos indica que las ceremonias en honor de un personaje de la élite (¿sacerdote ?, ¿jefe político-religioso ?) se efectuaban en plazole- 
tas circulares : por lo tanto, en espera de ulteriores investigaciones arqueológicas, podemos hipotetizar que tales plazoletas eran lugares ceremoniales.

Las complejas construcciones y movimientos de tierra realizados en PinchayRiway sin embargo nos dejan suponer que el sitio era probablemente una dependencia de un centro mucho más grande.

Al extender la comparación también al sitio de Joncopampa (Isbell 1989) (que comprende unos edificios construidos con muros parecidos a los muros sencillos de Pinchay-Riway pero que, según Isbell, pertenece más bien a la cultura Huari) se puede suponer que en el valle de Chacas, y precisamente en el sitio de Pinchay-Riway, la cultura Recuay podría haber perdurado más que en el Callejón de Huaylas.

La alta posición de Pinchay-Riway que, con el espolón amurallado domina el valle hacia Chacas, puede estar relacionada con motivos políticos, es decir defensivos, pero también podría tener algún motivo religioso. El sitio, rodeado por altos cerros y dos nevados, posiblemente se consideraba más cerca de los dioses que el valle de Chacas y es posible que ya en tiempos del Primer Período Intermedio se atribuyera a los cerros y a las rocas una dimensión sagrada al igual que los incas la harían, casi ocho siglos después. El lugar podía ser considerado entonces como sagrado a causa de su posición geográfica tanto para los vivos como para los difuntos. De todos modos, en el ámbito de los poderes teocráticos, como parecen haber sido los de las culturas precolombinas peruanas y también de la de Recuay, los límites entre las motivaciones políticas y las religiosas a menudo son imperceptibles.

\section{NOTAS}

1. Laura Laurencich Minelli, que dirige el proyecto, realizó la mapificación arqueológica del sitio y la redacción de esta nota, Alberto Minelli efectuó el trabajo topográfico y Carolina Orsini la recolección de superficie.

2. De cada sector se proporciona la altura media s.n.m. y, cuando hay mucho desnivel, también la altura máxima.

3. Del Pirushtu de Chacas procederían importantes monolitos grabados que se conservan en la Alcaldía, en el Colegio y en la Parroquia de Chacas.

4. La cara externa de las piedras gris-amarillento es aproximadamente rectangular y mide en promedio $20 \times 40 \mathrm{~cm}$.

5. Cf. Grieder 1978, fase Huacohú y Usú. El investigador norteamericano evidencia en particular para estas fases la progresiva desaparición de la pintura negativa, y la difusión de una cerámica anaranjada tosca con decoraciones en rojo, muy semejante a la que se encuentra en Pinchay-Riway. Subrayamos también las muchas comparaciones que se pueden hacer entre las formas de las vasijas, en particular con los bordes de cuencos (p. 69, fig. R, S, T) y de ollas (p. 71, fig. E, F, G, H).

6. Las medidas de la cámara funeraria son $4 \times 3 \times 1,20 \mathrm{~m}$ de altura, las medidas de la casita que la contiene son $4,40 \times 4,50 \times 2,50 \mathrm{~m}$ de altura.

7. Las medidas máximas de la pequeña cueva, antesala de la cámara funeraria, son : $2,10 \times 0,90 \times 1 \mathrm{~m}$ de altura.

8. La cámara funeraria es rectangular y mide $1,75 \times 1,45 \times 1 \mathrm{~m}$ de altura.

9. Como corona se entiende el espacio circular entre dos muros concéntricos. Las alturas de los muros se midieron a partir del piso antiguo interno.

10. En plano, estas pequeñas estructuras miden respectivamente $56 \times 30 \mathrm{~cm}$ y $40 \times 20 \mathrm{~cm}$. 


\section{REFERENCIAS BIBLIOGRÁFICAS}

Alvarez-Brun, F., 1970. - Ancash : una historia regional peruama, Villanueva, Lima.

Bonavia, D., 1991. - Perú. Hombre e historia, Ed. Edubanco, Lima.

BURger, R. L., 1993. - Emergencia de la civilización en los Andes : ensayos e interpretación, Lima.

Gambini, W. E., 1983. - Santa y Nepeña, dos valles, dos culturas, M. Castillo, Lima.

Gero, J. M., 1999. - « La iconografia Recuay y el estudio de género », Gaceta arqueológica Andina, 25, IDEA.

Grider, Terence, 1978. - The Art and Archaeology of Pashash, University of Texas Press, Austin \& London.

IsBELL, W. H. 1989. - « Honcopampa : was it a Huari administrative centre ? », in : R. M. Czinarno, F. M. Meddens \& A. Morgan (ed.), The Nature of Wari. A Reappraisal of the Middle Horizon Period in Perú, pp. 98-113, BAR International Series 525, Oxford.

Kauffmann Doig, F., 1993. - Perú, a cura di G. Ligabue, Erizzo Ed., Venezia.

Lanning, E. P., 1965. - «Current research (Highland South America) », American Antiquity, 31, pp. 140 y ss.

Lapiner, A., 1976. - Pre-Columbian Art of South America, Abrams, New York.

Larco Hoyle, R., 1975. - Perí, Nagel, Ginevra.

Lothrop, S. K., 1964. - Les trésors de l'Amérique précolombienne, Skira, Genève.

Lumbreras, L. G., 1974. - The peoples and cultures of Ancient Peru, trans. by B. Meggers, Smithsonian Institution Press, Washington D. C.

Ponte, V., 2000. — « Siglos VII-X : transformación social y política en el Callejón de Huaylas », comunicación al III Simposio Internacional de Arqueología, PUCP, 18-20 de agosto 2000, Lima.

Ravines, R., 1994. - Historia general del Perú, tomo II, Ed. Brasa, Lima.

Valladolid Huaman, C. M., 1990. - «Auquis Marca (Pueblo Viejo) », Boletín informativo, 4, pp. 10-11, Museo Arqueológico de Ancash, Huaraz.

WeGner S., 1990. - « La investigación científica sobre la cultura Recuay », Boletin informativo, 4, pp. 3-9, Museo Arqueológico de Ancash, Huaraz

—, 1994. - Cultura Recuay, Ed. Cordillera, Ancash.

Willey, G., 1971. - An introduction to American Archaeology, vol. II, South America, Prentice Hall Inc., Englewood Cliffs, New Jersey. 